\title{
Optimization of a Hybrid Community District Heating System integrated with Thermal Energy Storage system
}

\author{
Behrang Talebi ${ }^{1}$, Fariborz Haghighat ${ }^{*}$, Paul Tuohy ${ }^{2}$, Parham A. Mirzaei $^{3}$ \\ ${ }^{1}$ Department of Building, Civil and Environmental Engineering, Concordia University, \\ Montreal Canada, H3G 1 M8 \\ ${ }^{2}$ Mechanical and Aerospace Engineering, University of Strathclyde, UK \\ ${ }^{3}$ Department of Architecture and Built Environment, University of Nottingham, UK
}

\section{Abstract}

Evidence from a various research suggests that buildings hold a vital role in climate change by significantly contributing to the global energy consumption and the emission of greenhouse gases. Considering the trend of higher energy consumption in the building sector, it is important to influence this sector by decreasing its energy demand. District generation and cogeneration systems integrated with the energy storage system have been suggested as a potential solution to achieve such planned goals.

Unlike the older generation of the DHS, where the focus of the design was on minimizing the system heat loss, in $4^{\text {th }}$ generation DHS, achieving higher system efficiency is made possible by picking the optimal equipment size as well as adopting the appropriate control strategy.

Designers have adopted different design methods for selecting the equipment size, however, finding the optimal size is a challenging task. This paper reports the development of a simplified methodology (dynamic optimization) for a hybrid community-district heating system $(\mathrm{H}-$ CDHS) integrated with a thermal energy storage system by coupling the simulation and optimization tools together. Two, existing and newly built communities, have been considered and the results of the optimization on the equipment size of both communities have been studied. The results for the newly built community is later compared with the one obtained from the conventional equipment size methods whereas static optimization methods and potential size reduction with the conventional method has been obtained.

Keywords: Hybrid Community-District Heating System; Thermal Storage; Multi-Objective Dynamic Optimization; Load Prediction Method

\section{"Corresponding Author: Faribor.Haghighat@Concordia.ca}




\begin{tabular}{|c|c|c|}
\hline Variable & Description & Unit \\
\hline$A$ & Thermal Storage Exterior Area & $m^{2}$ \\
\hline$C$ & Cost & $£$ \\
\hline $\mathrm{Cap}_{T S}$ & Capacity of Thermal Storage & $m^{3}$ \\
\hline$C P_{w t .}$ & Specific Heat of the Fluid, Water & $\mathrm{kJ} / \mathrm{kg} \cdot \mathrm{K}$ \\
\hline E & $\mathrm{CO}_{2}$ Generation & $\mathrm{kg}$ of $\mathrm{Co}_{2}$ \\
\hline$E_{n, m}$ & Equivalent Emission Generated by Boiler $n$ at Year $m$ per Unit of Energy Generated & $\mathrm{kg} . \mathrm{CO}_{2} / \mathrm{kg}$.fuel \\
\hline $\operatorname{ExCap}_{m}$ & Extra Capacity of Boiler m & $k W$ \\
\hline$F C_{n, m}$ & Fuel Cost of Boiler $\underline{\mathbf{m}}$ at Year $\underline{\mathbf{n}}$ & $£$ \\
\hline$i$ & Annual Interest Rate & $\%$ \\
\hline$I C$ & Initial Cost & $£$ \\
\hline$I C_{m}$ & Base Cost of the Boiler m & $£$ \\
\hline$I E_{A u x}$ & $\begin{array}{l}\text { Equivalent Emission Generated by Imported Energy Year m per Unit of Energy } \\
\text { Generated }\end{array}$ & $\mathrm{kg} . \mathrm{CO}_{2} / \mathrm{kg}$.fuel \\
\hline$I N$ & Annual Income from Selling Energy to Off-Site & $£$ \\
\hline$L C_{m}$ & Linearized Cost of Boiler m & $£ / k W$ \\
\hline$L C_{T S}$ & Linearized Cost of Thermal Storage & $£ / m^{3}$ \\
\hline $\operatorname{Loop}_{D N}$ & Demand Side Loop & $k W h$ \\
\hline$M$ & Boiler Number & \\
\hline$N$ & Year Number & \\
\hline$\square_{C h .}$ & Charging Efficiency $=0.98$ & \\
\hline$\square_{\text {Dis.Ch. }}$ & Discharging Efficiency $=0.96$ & \\
\hline$O C_{\text {annual }}$ & Annual Operational Cost & $£$ \\
\hline $\mathrm{PRFF}_{n}$ & Primary Resource Factor of the Fuel & \\
\hline PRFIE & Primary Resource Factor of the Imported Fuel & \\
\hline$P W_{o c}$ & Present Worth of Operational Cost & $£$ \\
\hline$Q \_B L D G_{(t)}$ & Energy Required by the Buildings, Users, at Time $\underline{t}$ & $k W h$ \\
\hline$Q_{-} \operatorname{Gen}_{(t, n)}$ & Energy Generated by Boiler $\underline{\mathrm{n}}$ at Time $\underline{\mathrm{t}}$ & $k W h$ \\
\hline$Q_{-} \operatorname{Losses}_{(t)}$ & Energy Lost Through Distribution Network at Time $\underline{t}$ & $k W h$ \\
\hline$Q \_N e t_{(t)}$ & Net Energy Required by the Network at Time $\underline{t}$ & $k W h$ \\
\hline$Q_{-} T S_{C h(t)}$ & Energy Sent to Thermal Storage at Time $\underline{\mathrm{t}}$ & $k W h$ \\
\hline$Q \_T S_{D i s . C h(t)}$ & Energy Discharged From Thermal Storage at Time $\underline{t}$ & $k W h$ \\
\hline$Q_{T S . \operatorname{loss}(t)}$ & Energy Loss of the Thermal Storage at Time $\underline{t}$ & $k W h$ \\
\hline$T \_O A_{(t)}$ & Outdoor Temp. at Time $\underline{\mathrm{t}}$ & ${ }^{\circ} \mathrm{C}$ \\
\hline$T \_T S_{(t)}$ & Thermal Storage Temp. at Time $\underline{t}$ & ${ }^{\circ} \mathrm{C}$ \\
\hline$U$ & Overall Heat Transfer Coefficient of Thermal Storage & $W /\left(m^{2} \cdot K\right)$ \\
\hline$V$ & Volume of the Thermal Storage & $m^{3}$ \\
\hline$V_{A u x}$ & Amount of Imported Fuel Used to Generate a kWh of Energy & kg.fuel/kWh \\
\hline$V_{n, m}$ & Amount of Fuel Used to Generate a kWh of Energy & kg.fuel/kWh \\
\hline$\rho_{w t .}$ & Density of the Fluid, Water & $\mathrm{kg} / \mathrm{m}^{3}$ \\
\hline
\end{tabular}


Abbreviation

\begin{tabular}{cc}
\hline Abbreviation & Description \\
\hline \hline H-CDHS & Hybrid Community District Heating System \\
DHS & District Heating System \\
DHW & Domestic Hot Water \\
NTHU & Non-Typical High Usage \\
NTMU & Non-Typical Medium Usage \\
NTLU & Non-Typical Low Usage \\
TTCU & Typical Thermostat Control Usage \\
TMY & Typical Meteorological Year \\
MLCP & Mixed Linear Complementarity Programing \\
LCC & Life Cycle Cost \\
\hline
\end{tabular}

41

42

43

Major TRNSYS Components

\begin{tabular}{ccc}
\hline $\begin{array}{c}\text { Type } \\
\text { No. }\end{array}$ & Name & Representing \\
\hline \hline 700 & Simple Boiler with Efficiency Input (Modified) \\
659 & Auxiliary Fluid Heater with Proportional Control (Proportional Boiler) & Biomass Boiler \\
& Equa. 2 & Auxiliary Boiler \\
& Equa. 3 & Boiler House Controller \\
534 & Vertically Cylindrical Storage Tank with Optional Immersed Heat Exchanger & Network Controller \\
512 & Sensible Heat Exchanger With Hot-Side Modulation & \\
940 & Tank-less Water Heater & \\
977 & Variable Speed Pump & Circulation Pump \\
$604 a$ & Bi-Directional, Noded Pipe with Wall \& Insulation Mass \\
952 & Buried Single Pipe & Under Ground \\
682 & Load Imposed on a Liquid Stream & Distribution Network \\
\hline
\end{tabular}

44 


\section{Introduction}

As a major energy consumer, the building sector accounts for about $40 \%$ of the total energy consumption in North America and Europe, respectively [1]. Various countries prioritize the implementation of energy enhancement strategies in this sector to respect the Paris Climate Accord, COP21[2]. Such strategies have been applied at various levels, including energy production, conversion, and user-demand, but the most effective solution touches the higher level known as energy management [3].

A Hybrid ${ }^{1}$ Community-District Heating System (H-CDHS) is a unique type of energy management integrating thermal storage within its multi-source energy fed system. Two types of renewable sources exist in terms of availability a) intermittent sources such as wind and solar, and b) non-intermittent sources such as biomass and geothermal. For the intermittent sources, thermal storage can regulate the demand which could decrease the dependency on nonrenewable sources. However, for non-intermittent sources, thermal storage can appreciably improve the system performance and its efficiency in other ways such as peak demand shaving. [4].

The major design issue of the older district heating system (DHS) generations $\left(1^{\text {st }}\right.$ to $3^{\text {rd }}$ generation) was mainly high heat loss in the distribution network due to the high-temperature media $\left(100^{\circ} \mathrm{C}\right.$ and more) $[5,6]$. In this regard, the optimization focus was on enhancing the system efficiency by controlling the heat loss from the system and subsequently, improving the system efficiency. As a result, most optimization studies have focused on minimizing the system heat loss. However, the new generation DHS ( $4^{\text {th }}$ generation) operates at a lower temperature $\left(50-60^{\circ} \mathrm{C}\right)$, and hence achieving higher system efficiency is possible by adopting

1 The term hybrid, represent the use of multiple energy generation sources, renewable source (Biomass Boiler) and non-renewable source (Gas Boiler), used in the boiler house. 
appropriate control strategies and also through optimization of the equipment size $[7,8]$. Note that, designing the $4^{\text {th }}$ generation DHS based on the conventional design method, sizing the equipment based on the peak demand load, could lead to oversizing of the equipment and low system efficiency. Therefore, the adoption of an optimal approach (for cost, energy and environmental impact) to enhance the efficiency of the DHS while designing the $4^{\text {th }}$ generation DHS became a standard practice among designers.

Different optimization methods have been developed to improve H-CDHS efficiency and to reduce the system's emission footprint and the overall cost $[4,9]$. Among the existing methods, mathematical methods based on continuous or discrete variables (Figure 1) [3, 1012], generic algorithms [3, 13-15] and neural networks systems are the most implemented techniques for optimizing the DHS efficiency.

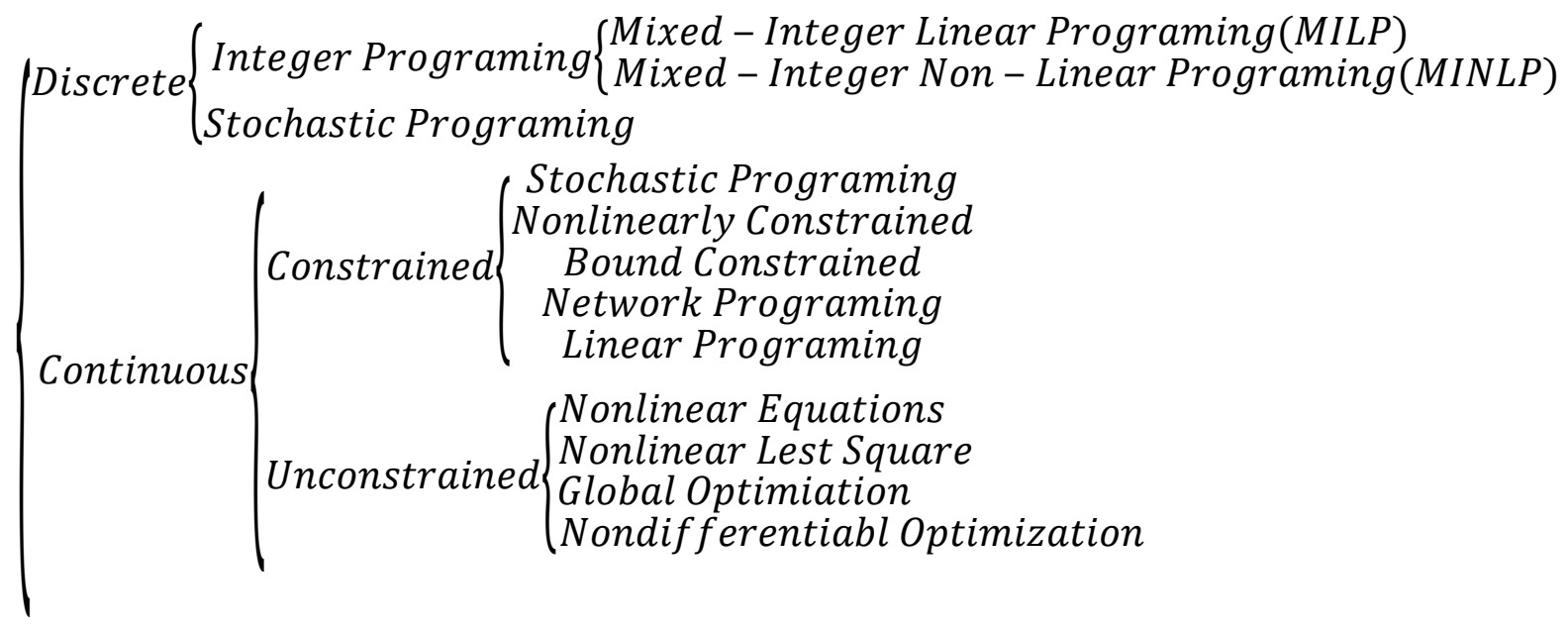

Figure 1: Summary of mathematical based optimization approaches

\subsection{Static and Dynamic Optimization}

Besides the mathematical approaches (as shown in Figure 1) adopted to formulate the optimization process, the optimization methods could be categorized either as static or dynamic optimization based on the dependency of the decision-making process with respect to time. In 
static optimization, the optimization time period remains the same for each iteration and the optimal solution is selected for a particular point of time within the given time period. In other words, in each iteration, regardless of any change in the optimization variables, the optimal solution is always at the same time. For example, static optimization obtains the optimal size of the equipment based only on the annual peak demand load. While in dynamic optimization, the optimization time horizon is split into a set of smaller time periods and the solution for each period affects the future solutions and possibilities. As a result, the optimizing agent takes into account this effect in the decision-making process.

Even though there is a scientific consensus on the mathematical definition of the static and dynamic optimization processes, there are many ongoing debates as to which type of optimization method should be used when it comes to use of the commercial energy simulation and optimization tools. Since similar simulation output could be obtained from all these commercial methods (e.g. energy demand profile), the interaction between the simulation and optimization tools can be used to identify the optimization type (static or dynamic optimization). For instance, in static optimization, the district component and the interaction between them are modeled either using the user-defined code or commercial simulation software $[19,20]$ in order to find the optimal size of the DHSs' equipment [11, 16-18]. Subsequently, the energy simulation is performed exclusively from the optimization process and a set of unique solution is obtained per simulation. In other words, the optimization population is generated by simulating the model over the simulation time period under different scenarios (optimizations variables) and the unique solution is obtained based on the objective function (i.e. cost and emission) under each scenario. Later on, the optimization tools use the unique solutions as an optimization population to find the optimized value of the objective function. It is worth mentioning that all unique solutions obtained from static optimization are for the same exact point of time (e.g. the peak demand time). By using the non-interactive 
model, i.e., separate simulation and optimization model (static model), there exists a higher

probability of decreasing the effectiveness of the optimization tool towards predicting the

121 optimal size of the equipment [16].

On the other hand, in dynamic optimization, instead of generating the optimization population by simulating the model for different scenarios, the optimization and simulation are carried out simultaneously. By simultaneously performing the optimization and simulation, not only a more comprehensive spectrum of the solution is generated as an optimization population, but also the generated off-spring population reflect the effects of previous hours. Due to the complexity of coupling the simulation and optimization tool in dynamic optimization, several 128 research works focused on the dynamic optimization using user-defined codes for system 129 modeling 2 [12, 21-23].

Since the dynamic optimization of the system using the detailed user-defined codes is

131 computationally expensive, and in many cases not feasible, different simplification approaches

132 have been adopted to decrease the computational time. These approaches resulted in a

133 simplification of the district energy model $^{3}$, using the reduced input file and the representative 134 weather or demand file for the design period instead of using the whole year profile, or the 135 combination of two. Considering the above-said research gap, the main objective of this study 136 is to develop a dynamic optimization platform that could explore the optimal equipment size 137 using the detailed demand profile in a timely manner. The developed model predicts the detailed 138 demand profile of the DHS and uses them along with detailed energy model of the DHS and 139 the equipment, and the interaction between them to dynamically optimize the entire system. 140 Subsequently, the optimal size of the equipment is obtained. The size of the equipment obtained

\footnotetext{
${ }^{2}$ Modeling the district components and the interaction between them.

${ }^{3}$ Represent the components and the interaction between them with a simplified equation
} 
141 from the model is later compared with the one obtained from the conventional method (design

142 day method), as well as using a static optimization tool, (Biomass optimization tool). In this

143 regard, data from an existing H-CDHS with an integrated thermal energy storage system is used

144 to optimize its boiler house to minimize its overall cost and $\mathrm{CO}_{2}$ emission.

\section{Methodology}

In this study, a mid-size H-CDHS considered earlier was used [24, 26]. The selected community is located in Cambuslang, Scotland and consists of 3 different types of residential buildings, newly renovated towers, newly built duplex detached houses and 4-story terrace buildings, with a total of $640+$ units. Multiple energy sources such as gas and wood pellets were used to provide the required energy to meet the heating and DHW demands. A well-insulated underground pipe network with a total length of $6 \mathrm{~km}$ (supply and return) is used to distribute

153 the energy between the generation and consumers. TRNSYS was used as the simulation platform to define the relationship between various system components and to couple the prediction and optimization tools. Also, a previously developed simplified load prediction model by the authors was used to dynamically predict the system demand load [24] . Results

157 obtained from the prediction tool (User Code) demand profile of the system, were fed as input 158 to the TRNSYS file in the text format. Adopting the predicted demand profile, TRNSYS model 159 determines the load required to be generated by the boiler house or to be stored in the thermal 160 storage by comparing the available stored energy and the predicted demand load.. Knowing the 161 net demand profile and the partial efficiency profile of each boiler, TRNSYS determines the

162 type and amount of the fuel required to offset the remaining demand. In the next step, the type 163 and amount of fuel as well required size of boilers and thermal storage are sent to the 164 optimization tool (GenOpt.) in form of an input file. Considering all the different possibilities, 
165 the optimization tool determines the optimal size of the equipment and overwrites the equipment size in the simulation tool, Figure 2.

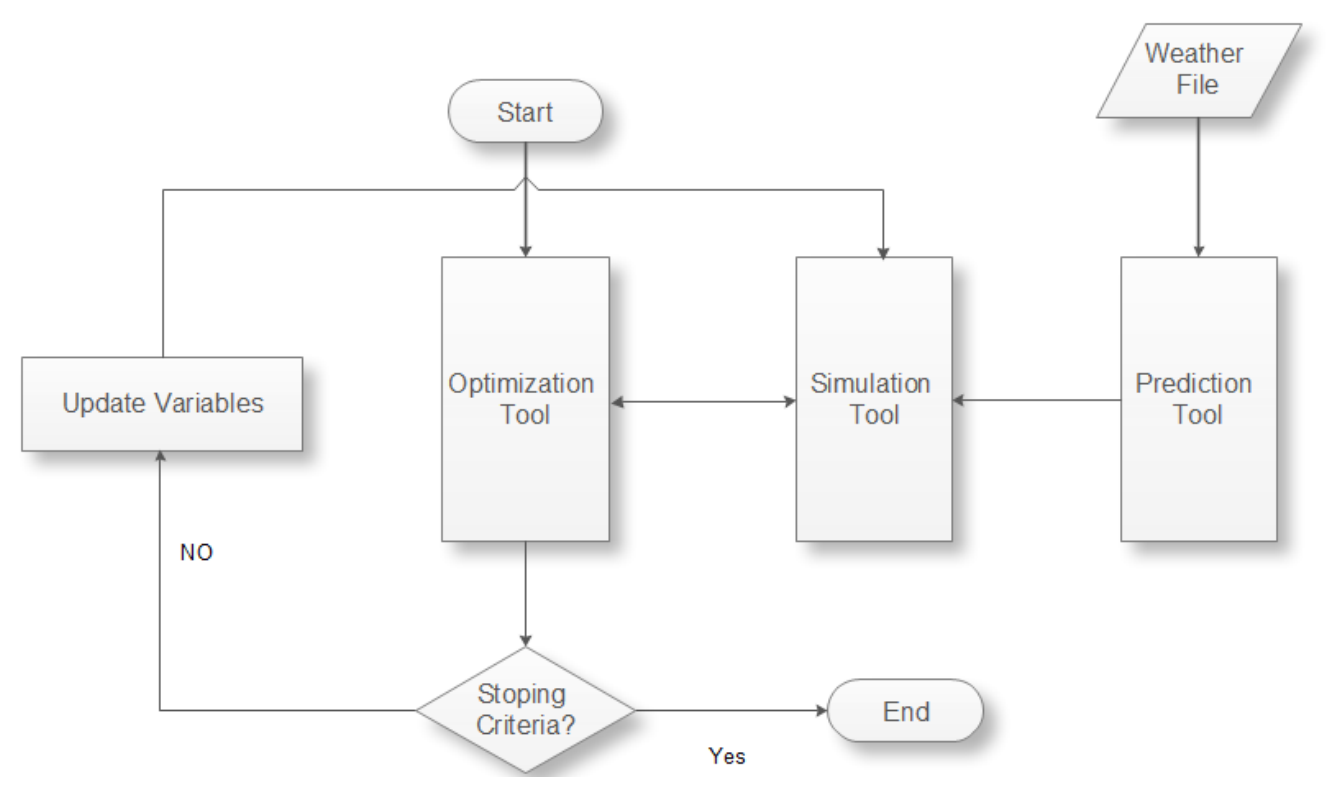

Figure 2: Prediction, Simulation, and Optimization Process Flowchart

\subsection{Load Prediction}

To optimize an H-CDHS, the first step is to predict the hourly energy demand profile of the entire H-CDHS, which includes the energy consumption and its corresponding losses. In general, there are three different techniques to obtain a community's energy demand profile: direct measurement, a comprehensive energy simulation tool used when data is not available, and simplified prediction methods in cases with high computational costs.

In this study, a simplified four-step procedure developed was used to predict the communities' energy demand profile [26]. The proposed model, by studying the energy behavior of the users, first, cluster the users into different groups, based on their energy consumption behavior. After segmenting the units among different clusters, the reference building for each cluster was obtained. In third step, using the energy consumption behavior of the reference building, the MLR model for each cluster was trained and used to predict the energy demand profile of the remaining unit within that cluster. The accuracy of the proposed 
procedure was validated using two different approaches, using both an inter-model comparison,

183 and comparing with measured data [26]. Using the validated model, the community demand 184 profile was predicted for two different scenarios:

- Scenario I: Optimizing the district's existing condition by considering users' demographic distribution regarding energy consumption habits.

- Scenario II: Optimizing the community as a newly built district by using design criteria and thermostat control to simulate all users' energy behavior.

Before performing the above-said optimization scenarios, in the first step, the community demand profile was predicted. In order to predict the community demand profile, occupants were divided into four different groups based on their energy consumption habits ${ }^{4}$.

192 The definition of each group and its contribution to the total population presented in more 193 detailed in [26]. Once these groups' energy consumption habits were available, the prediction model was trained using the proportion of each group within the community.

In the Scenario I, the proportion of the different occupants' type within the community remained constant and the results served as a basis of comparison for the optimization process.

197 Leaving occupants' demographic distribution untouched, the district energy demand profile for

198 Scenario I was predicted using the on-site weather data. Then using the on-site measured data

199 the accuracy of the energy simulation tools' (TRNSYS) was validated, as the all on-site 200 measured data correspond with this scenario. As a result, Scenario I compares the effect of 201 optimized equipment size and control strategy on energy consumption pattern of the existing 202 community, its $\mathrm{CO}_{2}$ emission, and cost.

\footnotetext{
${ }^{4}$ (Non-Typical High Usage (NTHU), Non-Typical Medium Usage (NTMU)), Non-Typical Low Usage (NTLU) and Typical Thermostat Control Usage (TTCU)) [26]
} 
204 weather and occupancy condition, both weather file and occupants' demographic distribution were replaced by the design condition. Hence in this scenario, the TMY3 weather file was used as a weather input data and, Typical Thermostat Control Usage (TTCU) profile was used as an occupancy profile. Note that the main difference between two scenarios is the energy behavior of the users. In newly built communities, due to unknown energy consumption profile of the users, the energy demand profile of the community was obtained based on the predefined schedules and the minimum temperature mandated by codes. However, in existing

211 communities, using the same procedure results in over estimating the energy consumption of 212 the community. In order to compare the effect of difference in energy demand profile on the 213 equipment size, boiler house under both scenarios has been sized and compared with each other. 214 As a result, in the first scenario, the existing community was sized by clustering the users and 215 adopting the actual energy behavior of them. However, in Scenario II, equipment has been sized 216 using the energy behavioral schedules and temperature mandated by codes, and subsequently 217 the obtained results were compared with the conventional method as well as static optimization 218 methods. Comparing the TMY3 file with the onsite measured weather data file used for 219 validating the model shows the average outdoor temperature of $9.3^{\circ} \mathrm{C}$ and $10.8^{\circ} \mathrm{C}$, and the minimum outdoor temperature of $-3.9^{\circ} \mathrm{C}$ and $-3.3{ }^{\circ} \mathrm{C}$ for $\mathrm{TMY} 3$ and onsite measured data, 221 respectively. Comparing the TMY3 average and minimum temperature, higher total load and 222 peak demand load are expected for both scenarios.

After obtaining both scenarios' typical usage behavior, a prediction model was trained based on the fraction of each community group's data. Figure 3, shows the design weather data, TMY3, and onsite measured weather data, while Figure 4 shows the demand heating 226 profile for these two scenarios. 


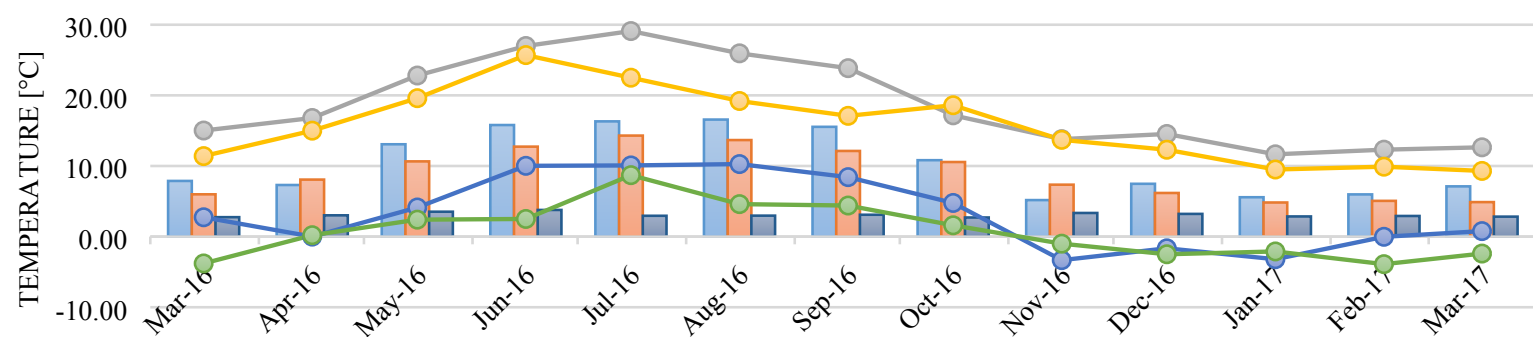

$\square$ Avg.Temp. M.D. $\square$ Avg. Temp. D.D. $\square$ Monthly Stdev. - - Max Temp MD

- - Max temp DD $\quad-$ - Min Temp MD $\quad-$ - Min Temp DD

Figure 3: Outdoor weather data (MD: Measured Data and DD: Design Data)

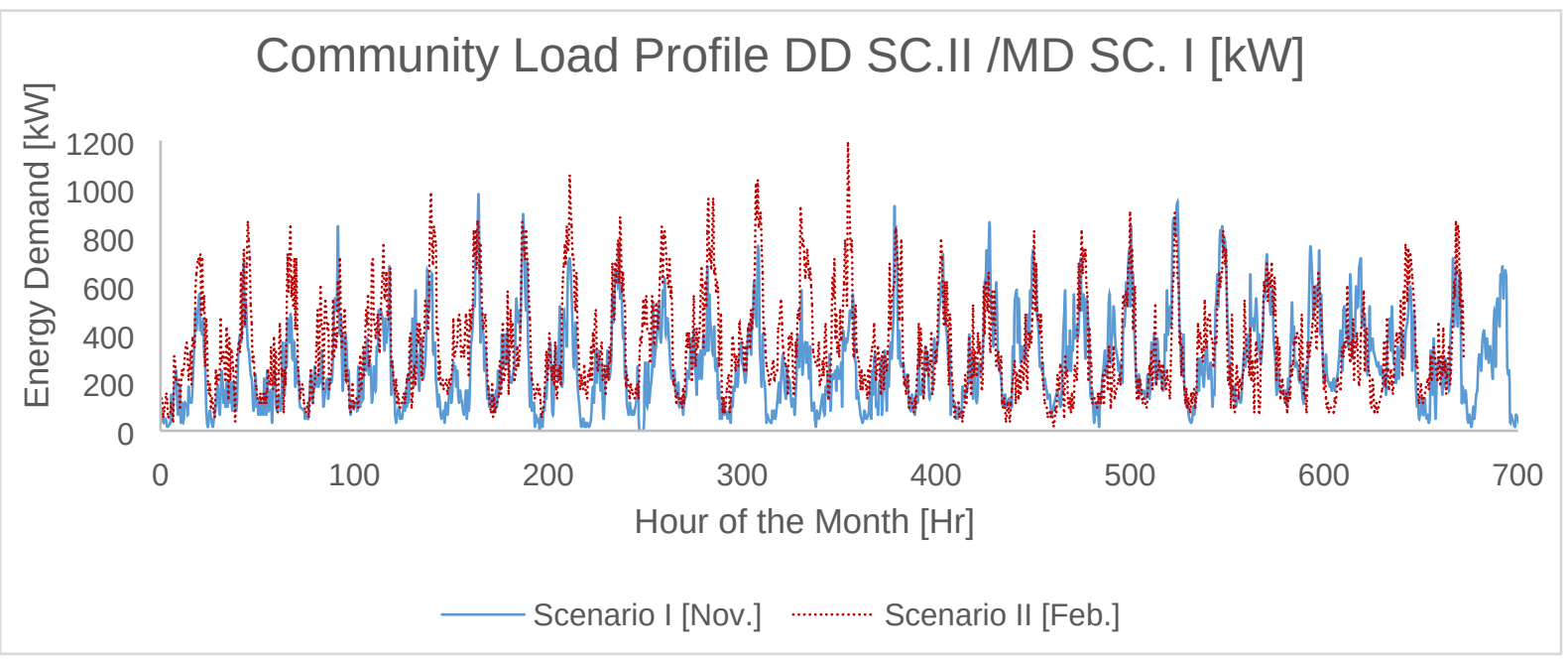

Figure 4: Predicted Demand Profile for Scenario I (November) \& Scenario II (February)

Figure 4 shows the heating demand profile of Scenario I \& II for the month when the

234 peak demand load occurred. The inference from the figure is that the peak-heating demand load 235 is $977.3 \mathrm{~kW}$ (2.8\% higher compared to the onsite measured data) in the Scenario I, and 1189 $\mathrm{kW}(25.1 \%$ higher compared to the onsite measured data) for scenario II. Note that, in

237 Scenario II, the entire community was simulated assuming all units were conditioned using the 238 thermostat control (TTCU). It is also important to note that domestic hot water usage was constant for both scenarios. Therefore, the $25.1 \%$ increase in peak demand load was associated only with the community's higher heating demand. 


\subsection{Energy Modelling}

242 TRNSYS was used to predict the district energy demand profile and the interaction between its 243 different components. To represent components, such as biomass boilers and building stock, 244 existing types in TRNSYS were modified. In general, TRNSYS has three major loops:

\subsubsection{Generation Loop}

The first loop (generation loop) consists of the auxiliary gas, biomass boilers, a

247 controller, and a heat exchanger, which feeds energy into the system, as shown in Figure 5 and

248 Figure 6. Since no specific biomass boiler type exists in TRNSYS, Type 700 was modified to 249 represent the biomass boiler by adjusting its efficiency, partial efficiency, and the control signal.

250 After adjusting the boilers' type, two controllers were assigned to the generation loop to adjust 251 the flow pattern between the generation/consumption loops and the storage loop. The first 252 controller compared the network's predicted demand load with the total capacity of the boiler 253 house and the need for the thermal energy storage system as a backup. The second controller 254 decides which boiler (biomass or gas) should operate to provide the required energy.

\subsubsection{Consumption Loop}

The consumption loop was constructed with Type 682, which represents the demand 257 profile of all units, (Figure 4). This Type reads the predicted demand profile through an external 258 link. The distribution network heat loss was modeled using Type 952.

\subsubsection{Storage Loop}

The storage loop was formed with two different configurations. The first configuration was modeled by simultaneously charging and discharging the thermal storage as shown in

262 Figure 5. 


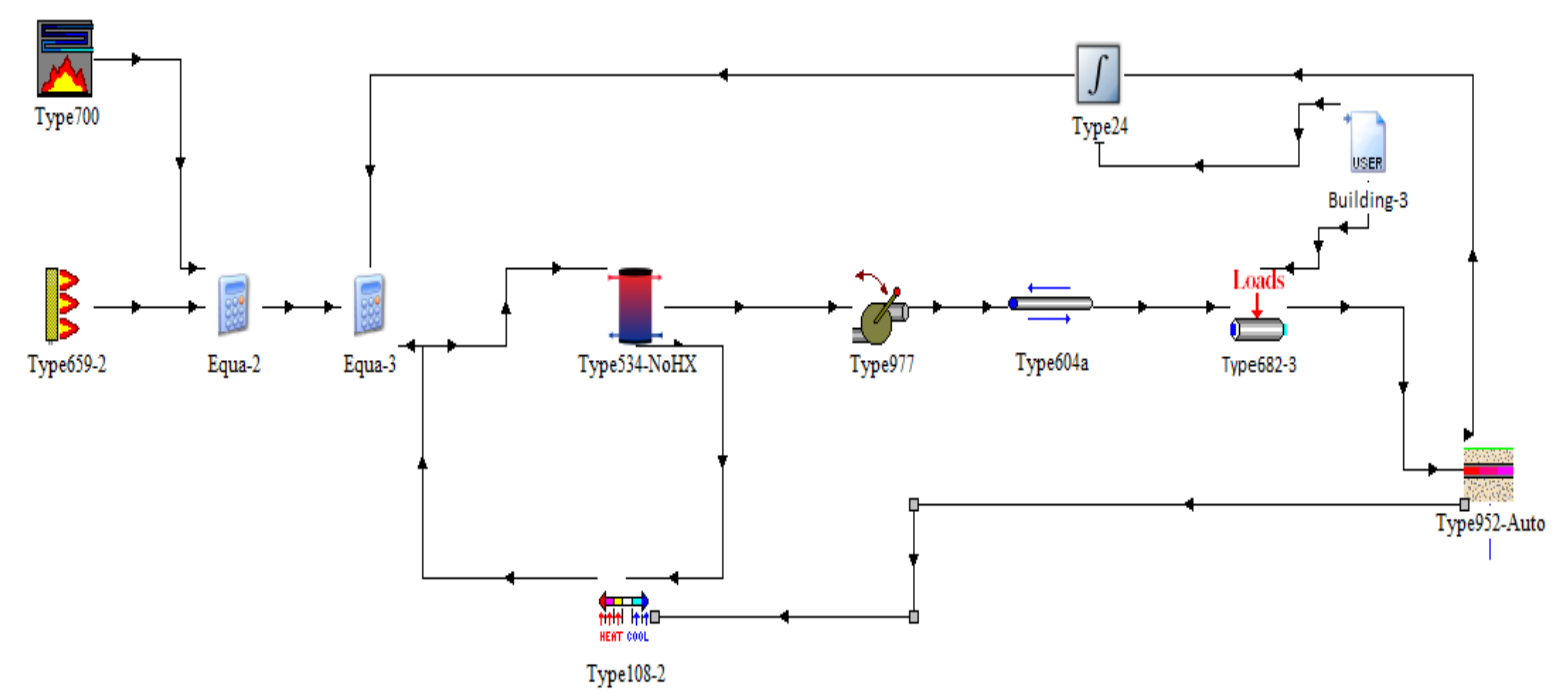

Figure 5: Simultaneous charging and discharging configuration

In other words, both the boiler house and distribution network was connected to the

267 thermal energy storage system. While the boiler house provided energy to the thermal storage 268 system, the latter supplied the energy to the distribution network. The second configuration was 269 modeled using a step-wise energy storing procedure (Figure 6). In this configuration, a controller monitored the direction to the thermal storage tank (either charged or discharged).

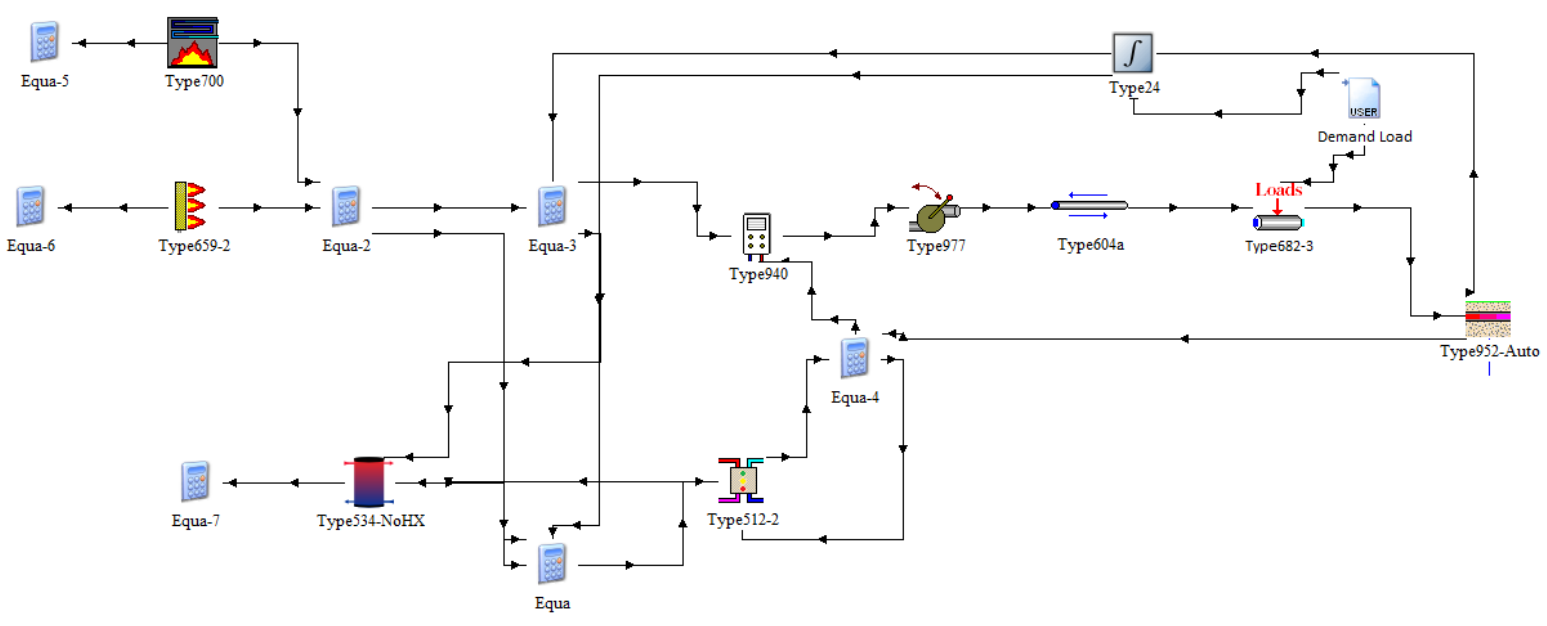

Figure 6: Step-wise charging and discharging configuration 
By comparing the preliminary results obtained from the total heat loss of the two 274 configurations (simultaneous and stepwise), it is inferred that the step-wise charging and 275 discharging configuration had the lower heat loss than simultaneous charging/discharging 276 configuration due to thermal system storage size and flow direction. Also, the step-wise 277 charging and discharging configuration has a higher overall energy efficiency compared with 278 the simultaneous charging/discharging due to on/off frequency of the generation loop in this 279 configuration (refer Figure 5 \& Figure 6). More detailed explanations regarding the efficiency 280 of the system are given in the following sections. As a result, the second configuration is used 281 as a base for optimization.

\subsection{Optimization Formulation}

As mentioned earlier in literature review, the main focus of this study is to optimize the size of the equipment in a dynamic manner. Existing method, such as using TRNSYS type 56 or Energy plus optimization function (both using GenOpt.) result in static optimization of the model. In both cases, the energy simulation is performed exclusively from the optimization process. Subsequently, an optimization population is generated by simulating the model over the simulation time period under different scenarios (optimizations variables) and the unique solution is obtained based on the objective function (i.e. cost and emission) for each scenario. The existing method is effective for optimization of the component which is not sensitive to previous time steps, such as boilers and earlier generation of the district system which does not have a thermal storage system. However, for components such as thermal storage systems which are sensitive to the amount of excessive/lacking energy at previous time steps, this method cannot result in finding the optimized solution. In this aspect, the existing method has been modified to perform the dynamic optimization.

296 For the design stage, a dynamic multi-objective optimization method was chosen to size the main components of the district network boiler house for the two defined scenarios. The model 
functions, life cycle cost (LCC) and $\mathrm{CO}_{2}$ emission. The optimization analysis focused on the on-site heat generation, but the option of purchasing auxiliary heating energy was also considered. This is because the primary goal of optimization is to size the main components of

302 the boiler house to minimize the investment and operational costs over a thirty-year cycle. To account for the effects of short-term load fluctuations on the components' optimal size, the optimization was conducted daily with an hourly temporal resolution. To improve model accuracy, other input data and model characteristics, including minimum and maximum output level constraints, and partial load efficiencies, were defined on an hourly basis. The system operational and fuel costs were also considered.

A controller type (Equa.-3 in Figure 6) was developed to compare the energy generated at each time-step with that in the boiler house (Equa.-2 in Figure 6) in accordance with the network demand load (Type 24) ${ }^{5}$ and flow direction. By comparing the demand load and generation capacity, controller fed the network first and then it decides whether to use the

312 disparity between generation and demand to charge or discharge the thermal storage system,

313 Equation 1-4. This implies that the controller regulates flow direction based on the general heat

314 balance equation, while other constraints (such as minimum operative temperature $\left(T_{-} T S_{(t)}\right)$ )

315 were set for the thermal storage (Equation 9) to ensure a minimum required temperature for 316 DHW usage:

$317 \sum_{n=1}^{N} Q_{-} \operatorname{Gen}_{(t, n)}+Q_{-} T S_{C h .(t)}-Q_{-} T S_{\text {Dis.Ch. }(t)} \geq Q_{-} N e t_{(t)}$

$318 Q_{-} \operatorname{Net}_{(t)}=Q_{-} B L D G_{(t)}+Q_{-} \operatorname{Losses}_{(t)}$

319 If

$$
Q_{G e n(t, n)} \geq Q_{N e t(t)} \rightarrow\left\{\left\{\begin{array}{c}
Q_{N e t}(t) \rightarrow \operatorname{Loop}_{D N}(t) \\
Q_{G e n(t)}-Q_{N e t(t)} \rightarrow Q_{T S_{C h .}(t)}
\end{array}\right.\right.
$$

\footnotetext{
5 Type 24 is the sum of heat loss of underground pipes obtained from Type 952 and the predicted demand load of the buildings obtained from the simplified method and fed to the TRNSYS model as an external user file (Demand Load)
} 


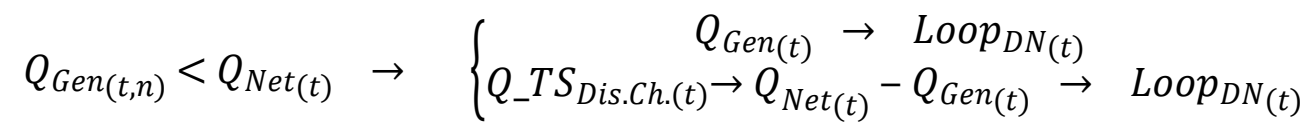

324 The equations used for modeling thermal storage, such as total energy at different time-steps

325 and boundary conditions applied to it, are as follows:

$$
Q_{-} T S_{(t)}=Q_{-} T S_{(t-1)}+Q_{-} T S_{C h .(t)} \cdot \eta_{C h .}-Q_{T S \operatorname{loss}(t)} Q_{T S \operatorname{loss}(t)}-\left(\frac{Q_{T S_{\text {Dis.Ch. }(t)}}}{\eta_{\text {Dis.Ch. }}}\right)
$$

$$
Q_{-} T S_{(t)} \geq 0
$$

$$
Q_{T S} \operatorname{loss}(t)=\left(T_{-} T S_{(t)}-T_{-} O A_{(t)}\right) \cdot U \cdot A
$$

$T_{-} T S_{(t)}=T_{-} T S_{(t-1)}-\left(\frac{Q_{T S} \text { Dis.Ch. }(t) / \mathrm{\eta}_{\text {Dis.Ch. }}}{V . C p_{w t .} \rho_{w t .}}\right)+\left(\frac{Q_{-} T S_{C h .(t)} \cdot \mathrm{\eta}_{C h .}}{V . C p_{w t .} \cdot \rho_{w t .}}\right)$

$T_{-} T S_{(t)} \geq 70^{\circ} \mathrm{C}$

After setting up the controllers, the optimization objective function (Equation 10) was

set up with the aim of optimizing the size of the biomass boiler(s) and thermal storage system, and minimizing the current net cost and $\mathrm{CO}_{2}$ emissions:

$$
\operatorname{Min}\{\operatorname{Obj}(C, E)\}
$$

where $C$ and $E$ are the cost and emission objectives. To make the objective function linear and to simplify it from $2 \mathrm{D}$ to $1 \mathrm{D}$, the optimization of was performed using the equation below:

$$
\operatorname{Obj}(C, E)=\alpha \cdot{ }^{C} / C_{0}+\beta \cdot \cdot^{E} / E_{0}
$$

where $\alpha$ and $\beta$ are the cost and emission importance factor in the final objective function. These factors were obtained based on the requirements/needs of the management board. Based on the

340 discussion with the community management office, the value of $\alpha$ and $\beta$ was considered as 0.75 341 and 0.25 , respectively. The cost associated function considers the entire C-DHS initial cost in 342 addition to the present worth of the life cycle operational cost. To define the initial cost 343 (Equation 12), the main boiler house equipment was divided into two modular modifiable parts 344 (boilers and thermal storage system) and fixed non-modifiable equipment (pumps and 
underground distribution pipelines). Note that, only the modular modifiable equipment cost was considered in the initial cost function and the initial cost of fixed non-modifiable equipment was excluded, as it remains constant regardless of the size of the modifiable equipment. For operational costs (Equation 13), the present fuel cost, the selling price of energy, and the buyout price of energy for surrounding houses for a 30-year period were considered using present worth method ${ }^{6}$.

$$
I C=\left(\sum_{m=1}^{N}\left(I C_{m}+L C_{m} \cdot \operatorname{Ex} \operatorname{Cap}_{m}\right)\right)+L C_{T S} \cdot \operatorname{Cap}_{T S}(12)
$$

where $I C$ is the linearized initial cost of the boiler house, ' $n$ ' is the number of years, FC is the 353 fuel costs of different boilers; ' $\mathrm{m}$ ' is the boiler number, $I N$ is the annual income from selling 354 heat to off-site users and $\mathrm{E}_{\operatorname{tax}}$ is the energy taxes. The initial investment cost includes the fixed and proportional variable expenses. The fixed component included the market value of the 356 smallest size of the equipment available on the market, $L C_{m}$, while the proportional cost was 357 determined by linearizing the extra cost associated with the higher capacity of the equipment, $358 L C_{m} \cdot E \operatorname{Cap}_{m}$. , Hereafter, in the text, $I C_{m}$ and $L C_{m} \cdot E x \operatorname{Cap}_{m}$ are presented as $\mathrm{A}$ and $\mathrm{BX}$, 359 respectively.

$O C=\left(\sum_{n=1}^{N} \sum_{m=1}^{M} F C_{n, m} \cdot(1+i)^{-n}\right)-\left(\sum_{n=1}^{N} I N \cdot(1+i)^{-n}\right)+\left(\sum_{n=1}^{N} \sum_{m=1}^{M} E_{t a x_{n, m} \cdot}(1+i)^{-n}\right)^{7}(13)$

361 The cost function (C) is the summation of the initial and operational cost, (Equation 14).

$$
C=I C+O C
$$

\footnotetext{
${ }^{6} P W_{O C}=O C_{\text {annual }} \cdot\left((1+i)^{n}-1 / i .(1+i)^{n}\right)$ where $\mathrm{i}$ and $\mathrm{n}$ are the annual interest rate and year number, respectively, and $\mathrm{OC}_{\text {annual }}$ is the annual operation cost.

${ }^{7}$ The energy discount rate (i) for Scotland is $0.9 \%$
} 
emission associated function was calculated using the following equation:

$$
E=\sum_{n=1}^{N} \sum_{m=1}^{M}\left(E_{n, m} \cdot V_{n, m} \cdot P R F E_{n}+I E_{A u x} \cdot V_{A u x} \cdot P R F I E\right)(15
$$

where $E_{n . m}$ represents the fuel emissions $\left(\mathrm{kg} . \mathrm{CO}_{2} / \mathrm{kg}\right.$.fuel) used for each boiler $(\mathrm{n})$ in a year $(\mathrm{m})$ of

367 the operation; $\mathrm{IE}_{\mathrm{Aux}}$ is the emission of the imported energy fed to the system from outside in 368 year, $\left(\mathrm{m}\right.$,) of the operation $\left(\mathrm{kg} \mathrm{CO}_{2} / \mathrm{kg}\right.$ fuel); $\mathrm{PRFE}_{\mathrm{n}}$ is the primary resource factor of the fuel; and

$369 V_{n, m}$ is the fuel volume used in each month ' $m$ ' by the boiler ' $n$ '. While calculating the costs,

370 the wood price was discounted in order to take into account the government incentive on the

371 price of wood pellets to encourage the small community to use biomass boilers. Note that values

372 of the primary energy factor for the wood pellets (PRFE) is 1.26 and for the natural gas (PRFIE)

373 is $1.2 .[27]$

To optimize the equipment size and to further minimize the overall costs, $\mathrm{CO}_{2}$ emissions over the life cycle, the first step is to define the price and emissions level for the different type of fuel. Table 1 represents the cost and $\mathrm{CO}_{2}$ values for wood pellets and natural gas as the main fuel type for the chosen district. Table 2 gives the initial cost of the major equipment.

Table 1: Energy cost \& emission for different fuel types

\begin{tabular}{ccc}
\hline & Emission $[\mathbf{k g ~ C O} / \mathbf{k W h}]$ & $\mathbf{f} / \mathbf{k W h}$ \\
\hline Wood Pellets & 0.039 & 0.061 \\
Natural Gas & 0.203 & 0.046 \\
Buyout & $\mathrm{NA}$ & 0.12
\end{tabular}

Table 2: Investment costs

\begin{tabular}{cccc}
\hline & Fixed $£[\mathbf{A}]$ & $£ / \mathbf{k W}[\mathbf{B X}]$ & $£ / \mathbf{m}^{\mathbf{3}}$ \\
\hline Wood Pellets Boiler & 125,000 & $362^{*}$ & NA \\
Gas Fired Boiler & 132,000 & $180^{* *}$ & NA \\
Wood Pellets Storage & NA & NA & 670 \\
Thermal Storage & NA & NA & 1,100
\end{tabular}




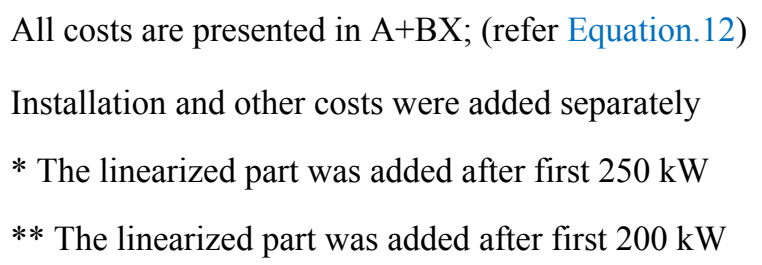

\section{Results}

As mentioned in Section 2.1, two different load scenarios were defined and served as a

383 basis of comparison within existing communities (Scenario I) or newly built communities

384 (Scenario II). Using the load demand profile for each scenario, the optimization process was applied separately, and the equipment's optimal size was determined.

\subsection{Scenario I (Existing Community):}

The Scenario I was defined based on the current situation of the H-CDHS regarding occupants' behavior. By keeping a similar occupancy distribution to that of a real case one, the potential annual cost saving and $\mathrm{CO}_{2}$ emission of the district over its life cycle was determined using the optimal equipment size and flow control (Table 3).

Table 3: Scenario I: Optimization results

Parameters

Peak Heating Load (kW)

Biomass Boiler (kW)

Auxiliary Boiler (kW)

Thermal Storage $\left(\mathrm{m}^{3}\right)$

Biomass Boiler Size Compared to the Peak Load (\%)

Coverage Percentage by Biomass and Thermal Storage (\%)
Existing Situation Scenario I

$\begin{array}{cc}1100 & 978 \\ 870 & 477 \\ 1300 & 609 \\ 50 & 16.3 \\ 79.1 & 49 \\ -- & 95\end{array}$

The optimization results for this scenario shows a significant reduction in boiler 
situation. Considering that only one boiler operates at a time, this fact only achieved by utilizing

a thermal storage system, which balances the demand and supply heat between the generation and consumption loops.

Comparing the optimized model results with field measurements show a dramatic drop 400 in $\mathrm{CO}_{2}$ emission (171.9 tons of $\mathrm{CO}_{2}$ /year or $23 \%$ ), as well as a considerable reduction in the total cost of the system $(79,056$ £/year or $17.6 \%)$. The cost and $\mathrm{CO}_{2}$ reductions are partially due to the lower efficiency of the oversized equipment working at a partial load while other parts can be associated to the non-optimal control strategy of the system and missing thermal storage.

Since specific weather data and occupants' behavior was considered in the Scenario I 405 (2016-17), the demand energy load of the community could change anytime based on the number of tenants or weather conditions. Consequently, after optimizing the system and 407 determining the optimal equipment size, the sensitivity of the design to any change in 408 community demand load due to change in the users' demographic distribution was determined. 409 To do that, two new cases (High and Low Usage) were defined. These newly defined cases 410 included a change in the fraction of occupants' types ${ }^{8}$ in the community compared with the 411 existing condition obtained from clustering results. In the High Usage Case, the fraction of 412 NTLU and NTMU users dropped, were added to the NTHU and TTCU users to represent a 413 higher demand load, see Table 4. In the Low Usage Case, the number of NTHU users dropped, 414 was added to the lower energy consumers such as NTLU and NTMU, see Table 4.

Table 4: Fraction of the occupants' types in different scenarios

\begin{tabular}{lccc}
\hline & Low Usage & Scenario I & High Usage \\
\hline \hline NTLU & $23 \%$ & $16 \%$ & $10 \%$ \\
NTMU & $39 \%$ & $24 \%$ & $15 \%$ \\
NTHU & $33 \%$ & $53 \%$ & $65 \%$ \\
TTCU & $5 \%$ & $5 \%$ & $10 \%$ \\
Peak Load $(k \boldsymbol{W})$ & 884 & 978 & 1,086
\end{tabular}

${ }^{8}$ NTLU, NTMU, NTHU, TTCU 
By changing the fraction of occupants, the energy demand profile for newly defined

418 cases was predicted and provided as input to the energy model (see Figure 6). The boiler house 419 equipment size remained similar to the Scenario I. After modeling these newly defined cases, 420 the system performance under new conditions was determined. Comparing the percentage of 421 the biomass boiler and thermal storage, which can cover the demand load of the community 422 between the Scenario I and High Usage Case (see Table 5), shows that in the High Usage 423 Case with 11\% higher peak, the percentage coverage time by biomass boiler dropped by $1.1 \%$.

424 Table 5: Performance of the optimized system under new demand profile load

\begin{tabular}{lccc}
\hline \multicolumn{4}{c}{ Sensitivity Results } \\
Parameters & Low Usage & Scenario I & High Usage \\
\hline \hline Peak Heating Load (kW) & 884 & 978 & 1086 \\
Biomass Boiler (kW) & 477 & 477 & 477 \\
Auxiliary Boiler (kW) & 609 & 609 & 609 \\
Thermal Storage (m ${ }^{3}$ ) & 16.3 & 16.3 & 16.3 \\
Biomass Boiler Size Compared to the Peak Load (\%) & 54 & 49 & 44 \\
Coverage Percentage by Biomass and Thermal Storage (\%) & 97.8 & 95.0 & 93.9
\end{tabular}

\subsection{Scenario II (Newly Built Community):} altered to the TTCU to represent the design criteria for newly built buildings. Table 6 presents

428 the optimal equipment sizes, resulting from the optimization of the boiler house for the

Scenario II.

Table 6: Scenario II: Optimization results

Parameters

Peak Heating Load (kW)

Biomass Boiler (kW)

Auxiliary Boiler (kW)

Existing Situation Scenario II

$\begin{array}{lc}1100 & 1189 \\ 870 & 661 \\ 1300 & 738\end{array}$


Thermal Storage $\left(\mathrm{m}^{3}\right)$

Biomass Boiler Size Compared to the Peak Load (\%)

Coverage Percentage by Biomass and Thermal Storage (\%)
50

79.1

$--$
32.8

56

98.8
432

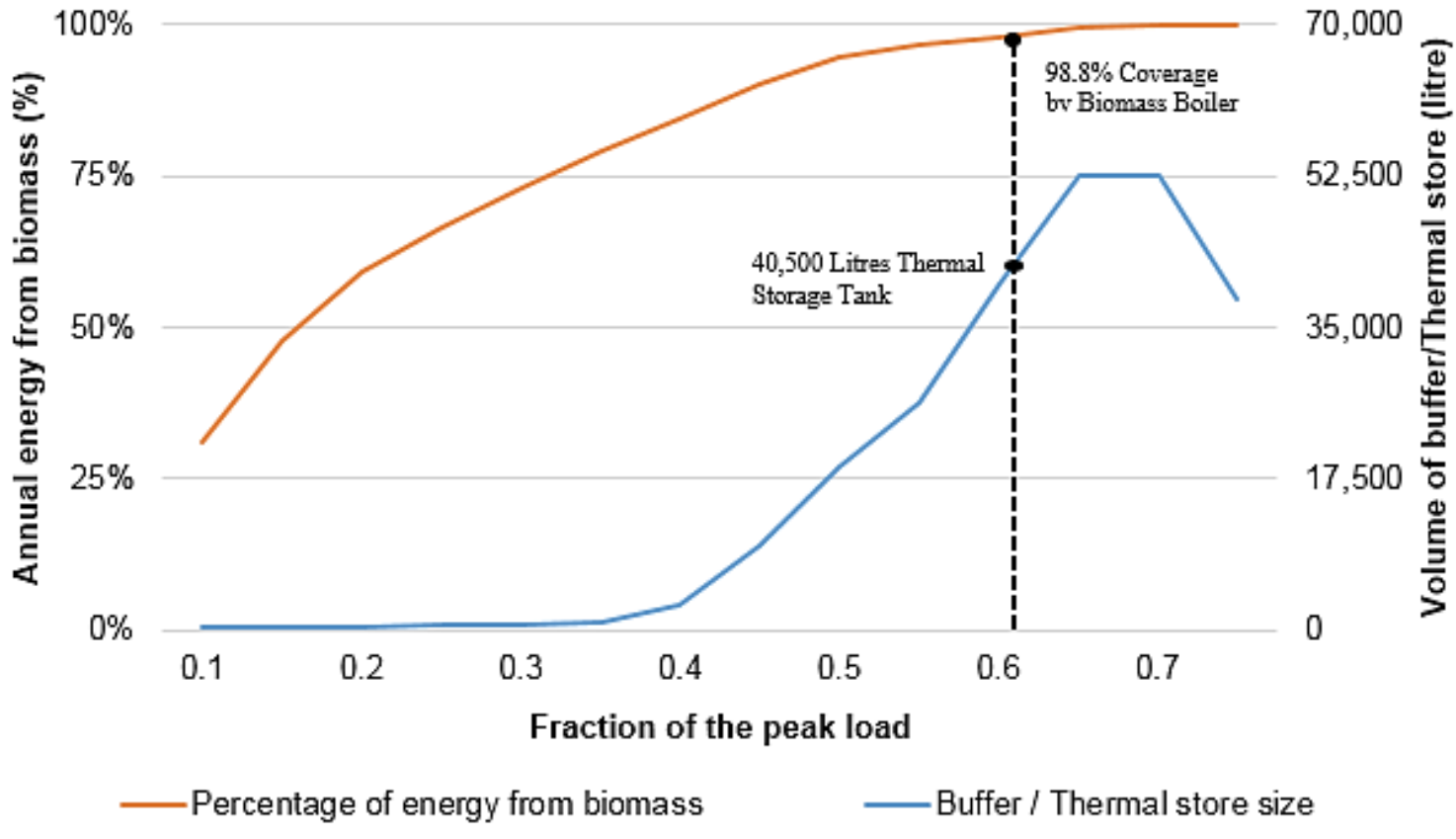

Figure 7: Optimal Equipment Size, Size of the biomass boiler as a percentage of a peak load for different annual \% of energy from a biomass boiler

Similar to the Scenario I, the capacity of the boiler optimal size, biomass and auxiliary boiler, used less than $60 \%$ of their capacity to respond to the peak demand load. In order to find the optimal size of the equipment using the static optimized sizing tools such as Biomass Boiler Sizing Tool (version 6.8.2), primarily the same annual biomass energy coverage (98.8\%) was determined. Using the same coverage percentage, the sizing tool suggests the biomass boiler with the capacity size of $62 \%$ of the peak load and $40.5 \mathrm{~m}^{3}$ thermal storage tank (refer to Figure 7). 
Table 7 presents the equipment size and cost associated with each design method.

445 
Table 7: Comparison of the equipment size, cost for different design strategies

\begin{tabular}{|c|c|c|c|c|c|}
\hline \multirow{2}{*}{ Technology } & \multirow{2}{*}{ Conventional } & \multicolumn{2}{|c|}{ Static Optimization Tool } & \multicolumn{2}{|c|}{$\begin{array}{c}\text { Proposed Dynamic } \\
\text { Optimization Process }\end{array}$} \\
\hline & & Size & $\begin{array}{c}\text { Size Reduction * } \\
{[\%]}\end{array}$ & Size & $\begin{array}{c}\text { Size Reduction * } \\
{[\%]}\end{array}$ \\
\hline Biomass Boiler [kW] & 870 & 737 & 15.3 & 661 & $24.0 \%$ \\
\hline Auxiliary Boiler [kW] & 1300 & 891 & 31.5 & 738 & $43.2 \%$ \\
\hline Thermal Storage $\left[\mathrm{m}^{3}\right]$ & 50 & 40.5 & 19.0 & 32.5 & $35.0 \%$ \\
\hline Cost $[\mathfrak{£}]$ & 734,440 & 602,224 & 18.0 & 538,372 & $26.7 \%$ \\
\hline
\end{tabular}

* Reductions calculated comparing with conventional method

Considering that only one boiler operates at a time, $98.8 \%$ coverage by biomass boiler was achieved using only thermal storage to balance between the generation and consumption loop. As shown in Table 7, this solution can reduce the size of both auxiliary and main biomass boilers into a fraction of their original size and, as a result, decrease the system heat loss while improving the district energy efficiency. The reduction in major equipment size of the district using the proposed dynamic optimization method caused a $196,068 £$ or $26.7 \%$ drop only in the system initial investment cost. Also, knowing the fact that the efficiency of the biomass boiler is lower when operated partially, two scenarios could be assumed for a non-optimal size equipment: 1) the biomass boiler works at its full capacity all the time while keeping the generation efficiency at maximum value; this can result in generation of an excessive amount of heat, which eventually is accounted as loss, and 2) the boiler works at partial load only to meet the network demand. This decreases generation efficiency due to the boilers lower partial capacity efficiency [25]. In both scenarios, the overall efficiency of the system drops.

\subsection{Impact of dynamic optimization in determining the operation period of the system}

As mentioned earlier in Section 1.1, the main difference between the static and dynamic optimization is in dependency of the decision-making process with respect to time. In other words, dynamic optimization, by breaking the demand profile into smaller periods and determining a solution for each period, considers the effects of demand at the previous hour on the optimal solution. Figure 8 (a), presents the charging/discharging profile of the thermal 
represents the thermal energy storage mean temperature and the district demand load.

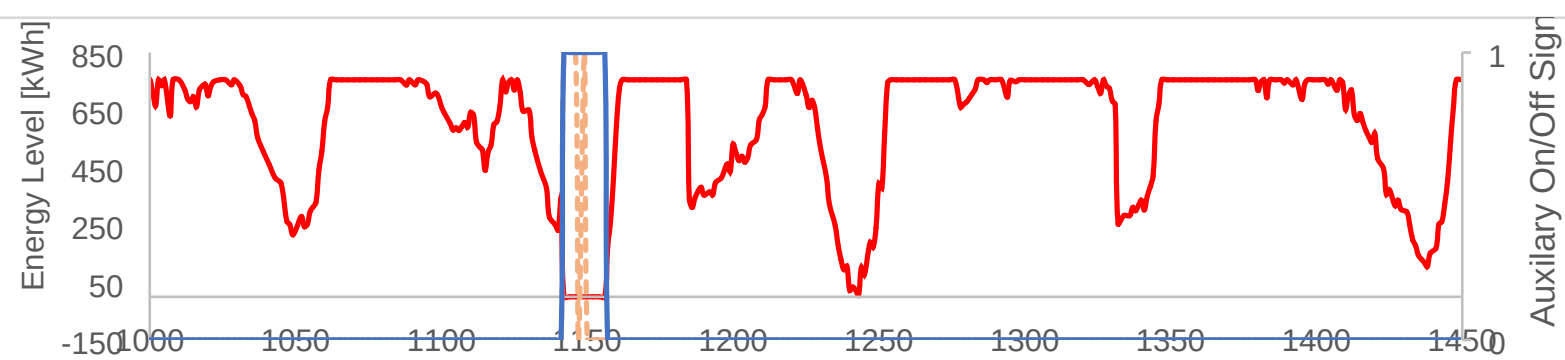

Time Counter

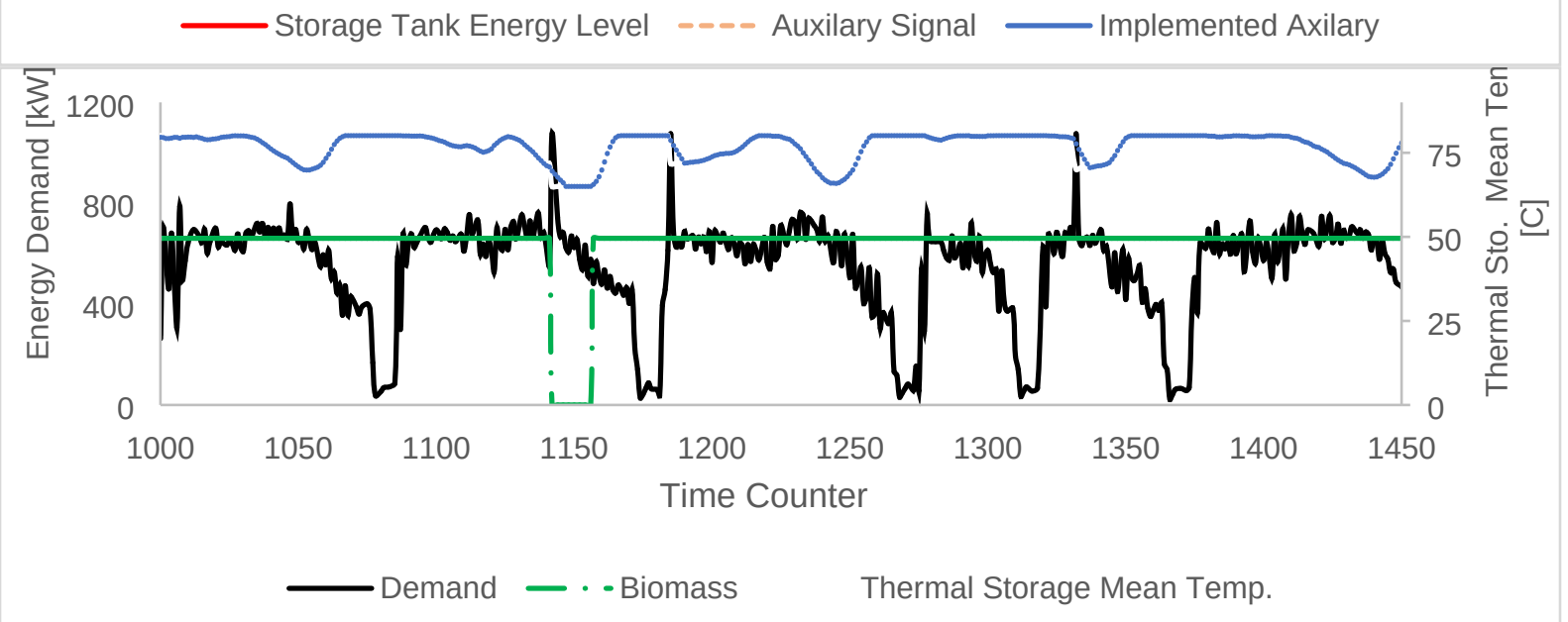

Figure 8: (a) Thermal storage energy level for a 10-day period in November; (b) Thermal storage temperature and district demand load for the same 10-days (Bottom)

In static optimization by only considering the peak demand in finding the optimal solution, the effects of the energy demand at previous hours on determining the optimal solution is neglected. On the other hand, in dynamic optimization, by considering the effects of the demand profile at a previous hour in determining the optimal solution can result in better utilizing of the thermal storage and lower size of the equipment. For instance, as presented in Figure 8, the response of the system to an identical demand varied based on the energy demand of the previous hours. In case of the first peak (shown in Figure 8 (b)), due to the high demand of the system prior to the peak, the thermal storage has been partially discharged, and as a result, the axillary energy is required to respond to the energy demand of the system. On the other 
hand, due to lower demand of the network prior to the second and third peak, the thermal storage

485 is fully charged, and no auxiliary energy is required.

Apart from determining the optimal size of the equipment, the optimal performance of

the system could be determined from the proposed dynamic optimization method. As shown in

Figure 8 (b), since the biomass boiler works constantly, the district demand load can be met

by a nominal size of the biomass boiler. However, when the demand load of the DHS is higher than the capacity of the biomass boiler, the deficit energy is met from the thermal energy

491 storage. On the other hand, when the demand load drops, the surplus energy is stored in the

492 thermal energy storage and the energy storage level swiftly increases. In peak demand period,

493 the instantaneous auxiliary system (gas boiler in this case), along with thermal storage, provide

494 required energy demanded by the district network since the biomass boiler cannot provide

495 enough energy for the system. Using this strategy while running the biomass boiler constantly

496 at full capacity for the optimized sized system, step-wise charging/discharging the thermal

497 storage can eliminate the need for the auxiliary energy $98.8 \%$ of the time while maintaining the 498 system's maximum overall efficiency.

\section{Conclusion}

This study proposes a novel optimization process called dynamic optimization for 501 existing and newly built communities by coupling optimization and prediction using a 502 TRNSYS based energy simulation platform. Optimization performed to calculate the overall 503 size of major energy generation and storing equipment, and operational control strategy for the community under different scenarios. In case of the existing community, Scenario I, comparing

505 the optimal equipment size with the existing non-optimal equipment sizes there exists a 506 considerable difference. The difference in equipment sizes (45\% smaller biomass boiler, 53\% 507 smaller auxiliary boiler and finally $67 \%$ smaller thermal storage size) between the existing 508 situation and the Scenario $\boldsymbol{I}$ is mainly due to the fact that the existing boiler house has been 
designed based on the conventional methods. Beside from the drop in the initial cost of the system $(267,716 £$ or $38.1 \%)$, the annual life cycle cost and $\mathrm{CO}_{2}$ footprint of the district also dropped by $79,056 £ /$ year or $17.6 \%$ and 171.9 tons of $\mathrm{CO}_{2} /$ year or $23 \%$ respectively. These

drops are due to the higher efficiency of the system operated at full capacity.

In case of a newly built district, Scenario II, three different design methods have been used to size the equipment, conventional, static commercial optimization tool, and the developed dynamic optimization process, and the respective results were compared. The results indicate that initial cost of the system using the proposed dynamic optimization method could drop by $26.7 \%$ compared with the conventional method while using the static optimization tool could only result in $18 \%$ drop in the initial cost of the system. These facts emphasized the importance of dynamic optimization of the system in order to achieve the real optimal solution.

\section{Acknowledgment}

The authors would like to express their gratitude to Concordia University for the support through the Concordia Research Chair - Energy \& Environment.

\section{References:}

1. Chu, S. and A. Majumdar, Opportunities and challenges for a sustainable energy future. Nature, 2012. 488: p. 294.

2. Christoff, P., The promissory note: COP 21 and the Paris Climate Agreement. Environmental Politics, 2016. 25(5): p. 765-787.

3. Zeng, J., J. Han, and G. Zhang, Diameter optimization of district heating and cooling piping network based on hourly load. Applied Thermal Engineering, 2016. 107(Supplement C): p. 750-757.

4. Talebi, B., et al., A Review of District Heating Systems: Modeling and Optimization. Frontiers in Built Environment, 2016. 2(22).

5. Wang, W., X. Cheng, and X. Liang, Optimization modeling of district heating networks and calculation by the Newton method. Applied Thermal Engineering, 2013. 61(2): p. 163-170.

6. Wang, J., Z. Zhou, and J. Zhao, A method for the steady-state thermal simulation of district heating systems and model parameters calibration. Energy Conversion and Management, 2016. 120: p. 294-305.

7. Chauhan, A. and R.P. Saini, Discrete harmony search based size optimization of Integrated Renewable Energy System for remote rural areas of Uttarakhand state in India. Renewable Energy, 2016. 94: p. 587-604. 
8. Mehleri, E.D., et al., A mathematical programming approach for optimal design of distributed energy systems at the neighbourhood level. Energy, 2012. 44(1): p. 96-104.

9. Olsthoorn, D., F. Haghighat, and P.A. Mirzaei, Integration of storage and renewable energy into district heating systems: A review of modelling and optimization. Solar Energy, 2016. 136: p. 49-64.

10. Bordin, C., A. Gordini, and D. Vigo, An optimization approach for district heating strategic network design. European Journal of Operational Research, 2016. 252(1): p. 296-307.

11. Zhou, Z., et al., Impacts of equipment off-design characteristics on the optimal design and operation of combined cooling, heating and power systems. Computers \& Chemical Engineering, 2013. 48(Supplement C): p. 40-47.

12. Ameri, M. and Z. Besharati, Optimal design and operation of district heating and cooling networks with CCHP systems in a residential complex. Energy and Buildings, 2016. 110(Supplement C): p. 135-148.

13. Fang, T. and R. Lahdelma, Genetic optimization of multi-plant heat production in district heating networks. Applied Energy, 2015. 159(Supplement C): p. 610-619.

14. Razani, A.R. and I. Weidlich, A genetic algorithm technique to optimize the configuration of heat storage in DH networks. 2016, 2016. 10: p. 12.

15. Barberis, S., et al., Thermo-economic analysis of the energy storage role in a real polygenerative district. Journal of Energy Storage, 2016. 5(Supplement C): p. 187-202.

16. Vesterlund, M., A. Toffolo, and J. Dahl, Optimization of multi-source complex district heating network, a case study. Energy, 2017. 126(Supplement C): p. 53-63.

17. Wang, H., et al., Optimization modeling for smart operation of multi-source district heating with distributed variable-speed pumps. Energy, 2017. 138(Supplement C): p. 1247-1262.

18. Rivarolo, M., et al., Design optimisation of smart poly-generation energy districts through a model based approach. Applied Thermal Engineering, 2016. 99(Supplement C): p. 291-301.

19. Magnier, L. and F. Haghighat, Multiobjective optimization of building design using TRNSYS simulations, genetic algorithm, and Artificial Neural Network. Building and Environment, 2010. 45(3): p. 739-746.

20. Asadi, E., et al., A multi-objective optimization model for building retrofit strategies using TRNSYS simulations, GenOpt and MATLAB. Building and Environment, 2012. 56: p. 370-378.

21. Campana, P.E., et al., Optimization of a residential district with special consideration on energy and water reliability. Applied Energy, 2017. 194(Supplement C): p. 751-764.

22. Schweiger, G., et al., District heating and cooling systems - Framework for Modelicabased simulation and dynamic optimization. Energy, 2017. 137(Supplement C): p. 566578.

23. Sameti, M. and F. Haghighat, Optimization approaches in district heating and cooling thermal network. Energy and Buildings, 2017. 140(Supplement C): p. 121-130.

24. Talebi, B., F. Haghighat, and P.A. Mirzaei, Simplified model to predict the thermal demand profile of districts. Energy and Buildings, 2017. 145: p. 213-225.

25. Camporeale, S.M., et al., Part Load Performance and Operating Strategies of a Natural Gas-Biomass Dual Fueled Microturbine for Combined Heat and Power Generation. Journal of Engineering for Gas Turbines and Power, 2015. 137(12): p. 121401-12140113.

26. Talebi, Behrang, et al., Validation of a community district energy system model using field measured data, Energy, 2018. 144: p. 694-706. 
27. R. Hitchin, U. Kingdom, K. E. Thomsen, and K. B. Wittchen, "Primary Energy Factors and Members States Energy Regulations," no. 692447, 2010 